\title{
AN INEQUALITY FOR THE RIEMANN-STIELTJES INTEGRAL ${ }^{1}$
}

\author{
RICHARD DARST AND HARRY POLLARD
}

ABstract. Let $g$ and $h$ be real valued and continuous on the interval $[a, b]$, and suppose that the variation, $V[h]$, of $h$ on $[a, b]$ is finite. By completely elementary methods, it is shown that $V[h] \cdot \sup _{a \leq \alpha<\beta \leq b}(g(\beta)-g(\alpha))$ is an upper bound for $\int_{a}^{b}(h-\inf h) d g$.

Several writers have recently obtained upper bounds for integrals of the form $\int_{a}^{b} h d g$, where $h$ is of bounded variation on the interval $[a, b]$ and $g$ is continuous there ([1], [2], [3, p. 573], [4]). It is our purpose to establish the following extension by completely elementary methods.

THEOREM. If $h$ is real and of bounded variation on the interval $[a, b]$ and $g$ is real and continuous there, then

$$
\int_{a}^{b} h d g \leqq(\inf h)(g(b)-g(a))+S[a, b] V[h],
$$

where $V[h]$ is the total variation of $h$, and

$$
S[a, b]=\sup _{a \leqq \alpha<\beta \leqq b} \int_{\alpha}^{\beta} d g .
$$

We observe first that it is enough to prove the inequality in the case inf $h=0$, when it becomes

$$
\int_{a}^{b} h d g \leqq S[a, b] V[h] .
$$

For the general case can be obtained from $\left(^{*}\right)$ by replacing $h$ in it by $h$-inf $h$. Clearly we may also suppose that for some $\xi, h(\xi)=0$. Since

$$
\int_{a}^{b} h d g=\int_{a}^{\xi} h d g+\int_{-b}^{-\xi} h(-x) d[-g(-x)]
$$

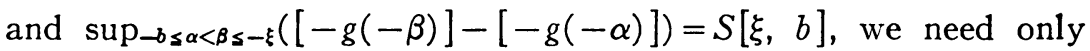
show

Received by the editors October 29, 1969 and, in revised form, November 14, 1969. AMS Subject Classifications. Primary 2646. bound.

Key Words and Phrases. Riemann-Stieltjes integral, bounded variation, upper

1 This research was supported in part by the National Science Foundation under grant no. GP 9470. 
(L) If $h \geqq 0$ and $h(b)=0$, then

$$
\int_{a}^{b} h d g \leqq S[a, b] V[h] .
$$

Proof of (L). Assume $g(a)=0$. Let $\phi(t)=\inf _{\xi \in[a, t]} g(\xi)$ and let $\psi(t)=g(t)-\phi(t)=\sup _{\xi \in[a, t]}(g(t)-g(\xi)) \leqq S[a, t]$.

Then $\phi$ is nonincreasing, $\phi(a)=0$, and $0 \leqq \psi(t) \leqq S[a, t]$. Moreover

$$
\begin{aligned}
\int_{a}^{b} h d g & =\int_{a}^{b} h d \phi+\int_{a}^{b} h d \psi \leqq 0+\int_{a}^{b} h d \psi \\
& =-\int_{a}^{b} \psi d h \leqq\|\psi\|_{\infty} V[h] \leqq S[a, b] V[h] .
\end{aligned}
$$

\section{REFERENCES}

1. T. Ganelius, Un théorème taubérien pour la transformation de Laplace, C. R. Acad. Sci. Paris 242 (1965), 719-721.

2. - An inequality for Stieltjes integrals, Proc. Fourteenth Scandinavian Math. Congress, Copenhagen, 1964.

3. P. Hartman, Ordinary differential equations, Wiley, New York, 1964. MR $30 \# 1270$.

4. R. Wüst, Beweis eines Lemmas von Ganelius, Jber. Deutsch. Math.-Verein. 71 (1969), 229-230.

Purdue University, Lafayette, Indiana 47907 